\title{
Ebola: SA has no outbreak 'laurels' to rest on
}

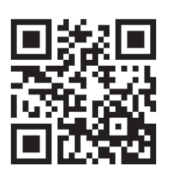

South Africa (SA)'s record in handling the initial HIV/ AIDS pandemic (without antiretroviral drugs) and the rapid spread of extensively drug-resistant tuberculosis (XDR TB) are major red flags warning that it may not have the capacity to face the deadly drug-defiant West African Ebola virus.

SA's daily TB death rate is nearly ten times the average daily Ebola death rate across the four affected West African countries from March this year - a sobering comparison when contemplating how we would handle an Ebola outbreak. Besides that, XDR TB kills $90 \%$ of its victims (multidrug-resistant (MDR) TB 50\% - about the same as a West African Ebola victim's survival chances).

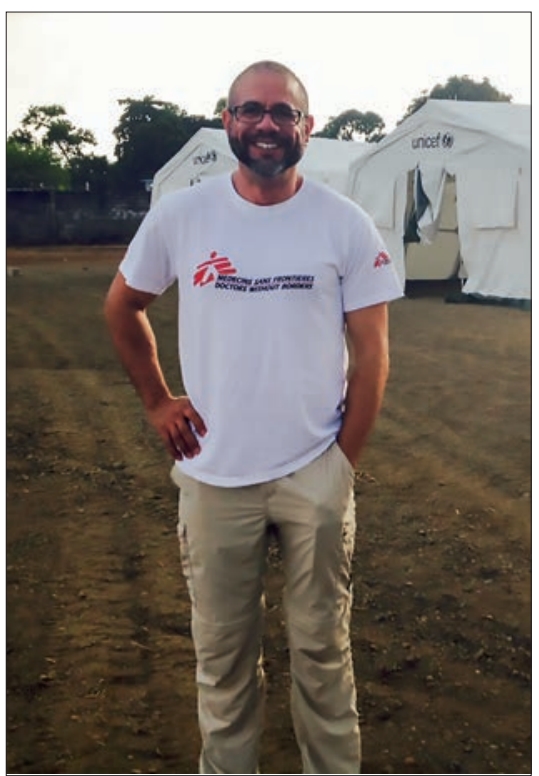

Dr Andrew Medina-Marino, local disease surveillance and laboratory systems expert, at a newly built isolation facility in Monrovia, Liberia, in August. Picture source: Dr Medina-Marino.

These analogies were drawn by an expert on disease surveillance and laboratory systems, Dr Andrew Medina-Marino, on his return to SA from Ebola-ravaged Liberia, one of the four West African countries across which the virus had claimed more than 1900 lives among 4000 suspected infections between early March and 3 September this year - and the pandemic is accelerating. A total of 2200 infections were confirmed in the woefully inadequate mobile laboratories, with hundreds more people certain to die, as what is justifiably one of the world's most-feared diseases has a nearly $50 \%$ current fatality rate. No Ebola cases have been confirmed among the hundreds of blood samples sent to the local National Institute for Communicable Diseases (NICD) for testing from throughout South African Development Community (SADC) member states, but the region is on high alert with emergency contingency plans for co-operation in detection, containment and awareness on 'fast-track', and a travel ban on all travellers from the affected countries in place. Because of SA and her immediate neighbours' geographical distance from West Africa - and the acute and severe nature of Ebola virus disease - officials claim it is 'highly unlikely' that cases will enter via land or sea. The highestrisk entry ports are OR Tambo International Airport in Gauteng and the nearby Lanseria Airport, from which all major medical air rescue company aircraft operate (two internationally). Travellers are being thermally screened at these airports and anyone with an elevated temperature questioned further, while OR Tambo has a modern emergency isolation/ transfer medical centre.

\section{SA's shaky track record} on drug-defying diseases A retrospective look at SA's XDR TB spread by Prof. Keertan Dheda, arguably the country's leading expert on drug-resistant $\mathrm{TB}$, at the National TB Conference in Durban in June, plus the lack of any communicable diseases regulation 6 years after the draft provisions were first published, add pinches of salt to ongoing public reassurances. Dheda, Professor of Medicine and Head of Pulmonology at the University of Cape Town (and one of the most published and cited TB academics in the country), estimates that several thousand local healthcare workers are currently TB-infected (the annual infection rate is $2-3 \%$ ), while 140 people die of all types of TB every day. Evidence that MDR TB is out of control is backed by known data; it has increased from 7350 notified cases in 2007 to 14161 in 2012. Dheda told Izindaba: 'We should be far more afraid of existing XDR TB than any fairly slim chances of an Ebola outbreak.'

While TB's incubation period and mode of transmission are very different to Ebola's (airborne spread v. bodily fluid and infected tissue spread), common denominators include infecting first-line primary healthcare workers, a dysfunctional public health system, low public awareness of basic infection control, and dismal, inappropriate education. At the Durban TB conference, Dheda made an impassioned plea for a nationally co-ordinated strategy to avoid the discharge of highly contagious 'therapeutically destitute' TB patients back into a careless void, with uniquely tailored solutions including home-based or community care, plus multidisciplinary teams in modernday sanatoria.

\section{If there's a local} outbreak, pray it's small and localised - expert Medina-Marino believes that general Ebola virus disease outbreak expertise and disease surveillance capacity in SA is probably insufficient to deal with 'anything more than a small, geographically contained outbreak. Senior Technical Advisor (Disease Surveillance and Laboratory Systems) at the local Foundation for Professional Development (FPD), he believes that a scattered West African-type Ebola outbreak in our townships could quickly turn into a public health nightmare. (The West African outbreak is the first in the world to reach urban areas.) Our government would have to lean heavily on the mainly US-funded NICD to roll out epidemiological and outbreak control programmes. Medina-Marino returned from a month of voluntary work in Ebola-ravaged Liberia early this August, where he gained invaluable experience and witnessed some of the rampant contagion first-hand.

\section{He believes that the widespread $S A X D R T B$ epidemic is analogous to the West African Ebola outbreak when it comes to comparing public health responses in the absence of any available medical intervention.}

His views, particularly about dysfunctional health systems, were echoed in principle by Prof. Sharon Fonn of the School of Public Health, University of the Witwatersrand, and co-director of the Consortium for Advanced Research Training in Africa, while Prof. Lucille Blumberg, head of the NICD's Surveillance and Outbreak Response Unit, warned that any Ebola outbreak would rapidly expose any existing deficiencies in a public health system, with poverty and fear aggravating contagion. With 150 healthcare workers dead among the more than 250 infected in the four (previously unaffected) West African outbreak countries, a graphic illustration of the 'fear factor' has already played out in SA. According to $\mathrm{Dr}$ Frew Benson, Chief Director of Communicable Diseases in the National Department of Health 


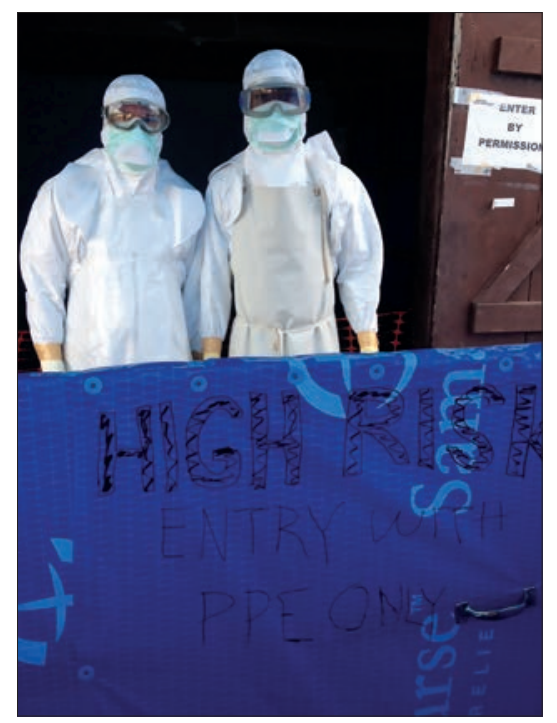

Beyond here lies death - without personal protection equipment. Picture source: Dr MedinaMarino.

$(\mathrm{NDoH})$, local nurses treating an already negatively diagnosed but heavily pregnant Ebola suspect refused to 'scrub in' for her caesarean section, doing so only under orders when told she was uninfected. 'You can imagine if we see more cases in SA, what the [caregiver] impact would be, he warned at an Ebola briefing at Wits University in late August.

\section{West Africa outbreak 'out of control'}

The severity of the epidemic is illustrated by one startling fact: the confirmed case tally on 3 September was 10\% more than all Ebola cases ever reported worldwide (2 000 people). Medina-Marino spoke to Izindaba after reading our report on the state of SA's Ebola prevention readiness, sharing his thoughts on interventions that would enable more effective detection and curtailment of any cases that might cross our borders. He is a veteran of the prestigious Centers for Disease Control in the USA, whose director Thomas Frieden warned on 2 September that 'the window is closing' on containing the West African epidemic, which he labelled 'a global problem'. Medina-Marino was seconded to the FPD, where he took a permanent job 3 years ago. He said there was 'no question' but that SA had among the world's best laboratory diagnostic capabilities, SA being one of only three countries asked by the World Health Organization (WHO) to send mobile diagnostic laboratories to West Africa. As one of the few doctors in SA with high-level outbreak response expertise, he volunteered to work cheek-by-jowl with colleagues from the world's uncontested Ebola outbreak veteran, Médecins Sans Frontières (MSF). MSF has 700 staff in Guinea, Liberia, Sierra Leone and Nigeria.
Lindis Hurum, MSF emergency co-ordinator for Liberia's capital, Monrovia, described the situation as 'catastrophic', with most of the city's hospitals closed and decomposing and highly infectious bodies lying in streets and houses. WHO Director-General Dr Margaret Chan declared the outbreak an international public health emergency, admitting that it was moving 'faster than we can control it.' The WHO is being severely criticised by a hugely overburdened MSF for its slow response (belatedly begun only after two deaths and seven confirmed cases in Lagos, Nigeria, the regional hub for international travel and business). Chan called for a global co-ordinated effort to combat what she termed 'the largest, most severe and most complex outbreak in the nearly four-decade history of this disease'. MSF President Dr Joanne Liu said her organisation was 'overwhelmed' and could now offer 'no more than palliative care', needing 800 additional beds for MSF's 160-bed Monrovian treatment centre alone. She said that rather than limit their responses to the potential arrival of an infected patient in their member countries, global health bodies 'should be helping save lives in West Africa'

\section{SA's poor track record in the early days of HIV and current XDR TB spread \\ Medina-Marino said that when it came to} disease outbreaks, SA had already clumsily faced the 'quite desperate' HIV pandemic (initially without antiretroviral drugs) and was currently failing to curtail a burgeoning XDR TB pandemic because of the lack of appropriate facilities and care to contain and prevent the highly infectious disease, for which no proven drug treatment exists. The country's response capacity (and expertise) remained focused on clinical care and treatment. When it came to identifying appropriate infection-risk factors and contact tracing, there was 'still a lot of work to be done'. In his opinion, only the mainly externally funded NICD was properly prepared to respond to an outbreak. Regardless of what the country's disease control officials said, SA did not have the capacity for fullscale investigations, especially for an outbreak anywhere near the scale of West Africa's. It could 'probably handle between one and three isolated cases.' 'When things actually happen, the system breaks down very fast', he said. 'All the money in the world' could not make up for delayed and unco-ordinated responses.

Prof. Fonn said that while SA's doctor-patien and nurse-patient ratios were better than those in the affected countries (SA has 8 doctors per 100000 population v. half a doctor per 100000 for Liberia and Sierra Leone, 1/100 000 for Guinea and 4/100 000 for Nigeria), there was no room for complacency. She called on the international community and countries to invest in the broader health system capacity and not respond to each event as if it were a crisis.

'Curricula for medical students, nurses and doctors are inadequate and inappropriate (one basic example is not being taught how to put on and take off personal protection equipment without becoming infected). We don't have systems for quick, effective communication between providers and tailored to the local burden of disease, should anything happen, let alone an Ebola outbreak, said Fonn. Public messaging campaigns should be conducted before any outbreak, to avoid spreading panic in the population, 'so that when it happens, you're reimporting an old message', she added. Simply responding to an epidemic was 'totally inadequate', she said, stressing that putting money into 'the most cost-effective' health programmes was an internationally and oft-repeated 'mistake'. 'What people don't understand is that the healthcare system is the sea upon which health programmes float. Over and over, we put money into the ships and not into the sea, she said.

Blumberg stressed that once Ebola reached the cities it became very difficult to contain, the main enemies being poverty, fear and dysfunctional health systems. She said that the NICD's mobile health laboratory in Liberia was 'extremely busy', with every second sample testing positive. Benson claims that 48 of SA's health districts have received Ebola prevention and awareness training, while defence forces throughout the SADC region would be called in to curtail movement should an outbreak occur. Ebola-ready hospitals had been designated in every province, including one private sector hospital and two military hospitals (Medina-Marino questions how properly equipped they are). With the paucity of institutional training, basic healthcare worker protection training was being stepped up, while immigration officials were receiving twice-weekly updates and training and broader multisectoral awareness was being accelerated.

\section{Vicious 'circle of infection'}

Benson said that strengthening SA's screening capacity and facilities was essential. 'An outbreak becomes a vicious circle; once the health system becomes affected it feeds into the outbreak itself,' he admitted.

Asked what basic precautions should be taken by SA healthcare workers, MedinaMarino said that simply wearing gloves and asking patients whether they'd recently been in West Africa or in contact with someone from there would generally suffice. He challenged Benson's contention that people with 
Ebola were usually 'very sick' and typically presented to a hospital. In Medina-Marino's experience one of the first places a person with Ebola symptoms went to was a primary or community health clinic (all 250 West African healthcare workers were infected in this way). Here doctors and nurses were (initially) 'not necessarily thinking this person has Ebola' and interacted freely with patients, posing a major infection risk. Medina-Marino emphasised that there was 'no need to panic', but cautioned that there was 'good reason' for the SA government to be better prepared.

\section{Because of SA and her} immediate neighbours' geographical distance from West Africa - and the acute and severe nature of Ebola - officials claim it is 'highly unlikely' that cases will enter via land or sea.

His 'don't panic' message was echoed by $\mathrm{Dr}$ Charl van Loggerenberg, Regional Medical Director of International SOS (a 24/7 corporate medical assistance company that also includes the Air Rescue Africa air ambulance operation), who spoke to Izindaba on his return from an $\mathrm{NDoH}$ consultation of all relevant SA role players in the public and private sectors in Gauteng on 22 August. He revealed that the SA travel ban on passengers coming from any of the affected West African countries would not apply to medical air rescue companies, which already implemented the highest possible preand in-flight viral haemorrhagic fever (VHF) safety protocols and compliance with port health procedures for all clients. Van Loggerenberg said that the SA government wanted to use the global expertise of companies like his and Netcare 911 to help gather information and disseminate the best possible clinical pathways

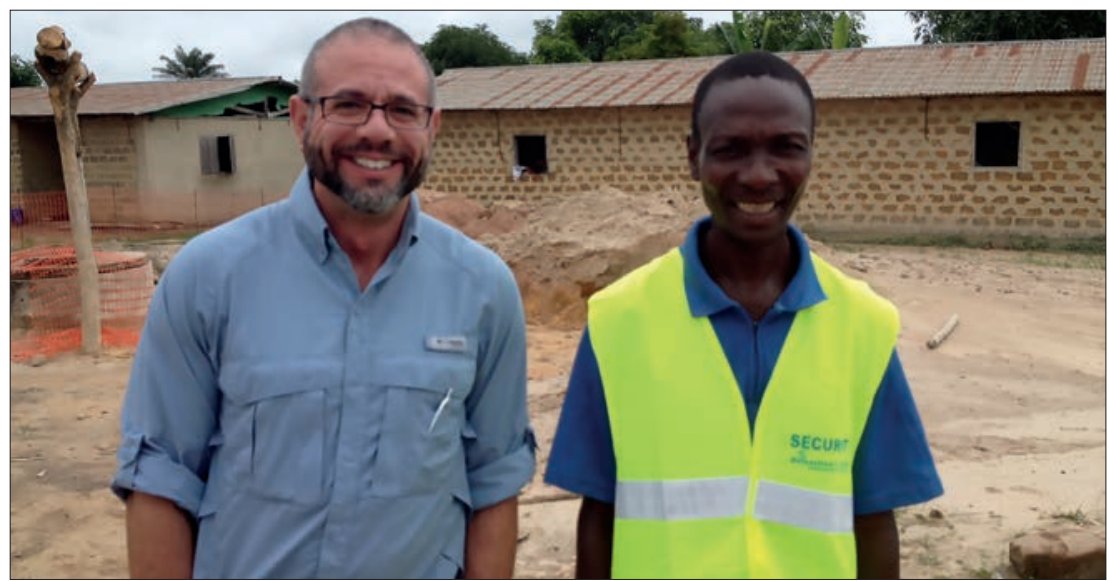

Dr Medina-Marino with a volunteer helper and Ebola survivor (he presented early). Picture source: Dr Medina-Marino. and emergency health worker precautionary guidelines. He said that general and nursing practitioners in border areas were the 'world's best gatekeepers', warning, however, that they should take robust travel histories from any patient presenting with fever associated even with mundane complaints such as toothache or earache. Netcare has developed a set of comprehensive clinical pathways that are used routinely as a precautionary measure by its emergency medical staff, doctors and hospital staff to assess any patient exposed to risk factors associated with VHF.

Asked why he thought the situation in Liberia had deteriorated so quickly, Medina-Marino said the civil war had 'decimated' its' health infrastructure, leaving the government starved of resources to deal with the scale of the outbreak. Another aggravating factor was sociocultural: because of the war, communities distrusted any government intrusion, refusing health authorities permission to remove dead bodies. In Liberia's war-ravaged northern Barkadu district, communities actually barricaded themselves. Health officials, only admitted after a fortnight, discovered 20 bodies and 15 people near death. 'Basically they didn't believe Ebola was real and only called for help when they were scared witless. Similar stories abound in Sierra Leone and Guinea, he said. Another major issue was the unprotected washing, embalming or dressing of bodies as part of the pre-funeral rites. At burials many mourners also touched the body as a 'final farewell'. 'How do you tell these communities to stop their traditions?' he asked. Another tragic and almost unavoidable problem was Ebola-infected children - if a child is vomiting or sick, how do you tell a mother or father not to touch them?

\section{Ebola spread in war-torn Liberia 'out of control'}

By the time Medina-Marino left on 9 August, the situation in Liberia was 'completely out of control'. He said that the unique and unprecedented nature of the current widespread, urban West African outbreak (previous outbreaks in rural East Africa had been geographically 'clustered') meant there were 'no models to deal with this'. He believes that the widespread SA XDR TB epidemic is analogous to the West African Ebola outbreak in terms of comparing public health responses in the absence of any available medical intervention.

Both Benson and Blumberg agreed that healthcare workers were at greatest risk. Ebola, the onset of which is sudden and severe, is only infectious when its carrier is symptomatic and ill (and for some time after death). It typically only spreads via contact with bodily fluids and infected tissue. These include blood, vomit, faeces, sweat, saliva, tears, urine and (least likely, though feasible) semen. Unprotected home care settings, low hygiene awareness and the tradition of washing the newly dead mean that it wipes out entire families and communities, with the spread amplified in hospitals with poor infection control practices.

\section{Thermal scanning more} political than pragmatic She said that thermal scanning at airports was relatively ineffective, as somebody incubating the virus could be asymptomatic. Its 'yield' was infinitesimally low, and it was far more political than pragmatic. Meanwhile, the SADC ministers of health plus the regional SADC and WHO officials held an emergency meeting in Johannesburg on 6 August to collaborate on critical Ebola detection, prevention and control strategies, releasing a lengthy statement of intent.

Izindaba has established that the four known Ebola cases air-lifted to the USA and Spain for treatment (one died, three were 'improving') were foreign healthcare workers and/or missionaries. Their treatment on home soil with the promising but experimental drug Z-Mapp (and that of a select few West African patients) sparked controversy, given that more than 1900 Africans have died without access to existing (low) stocks. However, medical ethicists pointed to Z-Mapp's untested nature, saying that public health measures should be paramount.

At the time of writing, the Ebola death toll in the then newly affected Democratic Republic of Congo had reached 31, albeit from a different strain to that in West Africa.

Chris Bateman

chrisb@hmpg.co.za

S Afr Med J 2014;104(10):653-655. DOI:10.7196/SAMJ.8894 